\title{
Brain Herniation:
}

\section{A Revision of Classical Concepts}

\author{
C. Miller Fisher
}

\begin{abstract}
This paper is an update on evolving ideas about brain herniations. Following observations on cerebellar pressure coning that raised doubts about its reputed lethal connotations, herniation at the tentorium was re-examined for its role in critically damaging the brain stem. Combining clinical, pathologic, computed tomography and magnetic resonance imaging data, it is concluded that temporal lobe herniation is not the means by which the midbrain sustains irreversible damage in acute cases, but rather lateral displacement of the brain at the tentorium is the prime mover and herniation a harmless accompaniment. Transtentorial herniation has been investigated with computed tomography using the three calcification relationship and descent through the tentorial opening could not be documented. Bilateral brain stem compression in acute bilateral cases must be distinguished from herniation. Upward cerebellar herniation is only the sign of an overfull posterior fossa. Subfalcial herniation is tolerated unless lateral displacement is excessive.
\end{abstract}

RÉSUMÉ: Revue des concepts classiques touchant la hernie de tissus cérébraux. Cet article est une mise à jour de concepts en évolution sur la hernie de tissus cérébraux. A la suite d'observations qui ont jeté des doutes sur sa connotation létale, nous avons réexaminé les conséquences de la hernie de tissus cérébelleux au niveau de la tente du cervelet. En combinant les données cliniques, anatomopathologiques et d'imagerie par tomodensitométrie et résonance magnétique, nous concluons que la hernie du lobe temporal n'est pas la cause du dommage irréversible du tronc cérébral dans les cas aigus, mais bien le déplacement latéral du cerveau au niveau de la tente du cervelet et que le tissu hernié n'en est qu'une conséquence inoffensive. Lors de l'étude de la hernie transtentorielle par tomodensitométrie utilisant trois points de repère osseux, la descente de tissus à travers l'orifice tentoriel n'a pas pu être documentée. La compression bilatérale du tronc cérébral dans les cas aigus bilatéraux doit être distinguée de la hernie. La hernie cérébelleuse vers le haut n'est qu'un signe d'une fosse postérieure trop remplie. Ces hernies sont bien tolérées, à moins qu'il n'y ait un déplacement latéral excessif.

Can. J. Neurol. Sci. 1995; 22: 83-91

The several sites of brain herniation that complicate expanding intracranial processes were originally described in the first half of this century - cerebellar pressure cone at the foramen magnum,' uncal herniation at the tentorium, ${ }^{2}$ transtentorial herniation, ${ }^{3}$ subfalcial herniation, ${ }^{2}$ and upward cerebellar herniation. ${ }^{4}$ Use of these terms, clinically and pathologically, with their accompanying inferences, is deeply ingrained. In the great majority of instances, the observations and the deductions therefrom were based on the findings at autopsy in cases of brain tumor, where the clinical picture had evolved over a period of weeks or months.

Observations in cases of acute hypertensive cerebellar hemorrhage raised doubts about the accepted views of cerebellar pressure coning. ${ }^{5}$ In turn, temporal lobe herniation came in for re-examination, ${ }^{6}$ especially as it related to cases of acute unilateral or bilateral expanding hemispheric mass lesions. Here also, evidence is accruing which suggests that herniations are not special mechanisms by which the brain stem is damaged, but may be late irreversible end-stage phenomena mainly of interest to the pathologist. Clinically, emphasis should be focussed on the earlier stages of events in the region of the tentorium. Conceivably, modern surgical management would benefit were the pathophysiologic dynamics more precisely known.

The object of the present paper is to offer alternatives to the classical thinking about herniations. Personally, the subject became of interest some 25 years ago and a chronological account should best serve to trace the development of ideas in an orderly fashion.

\section{Cerebellar Pressure Cone}

In a pathological study of 18 cases of acute hypertensive cerebellar hemorrhage that had run a fatal course, ending in

\footnotetext{
From the Neurology Service, Massachusetts General Hospital. Boston. RECEIVED AUGUST 26. 1994. ACCEPTED IN FINAL, FORM DECEMBER 13, 1994. Reprint requests to: CM Fisher, M.D.. Neurology Service, Massachusetts General Hospital, Boston, MA USA 02114
} 
posterior fossa brain stem compression and respiratory failure, 17 showed no cerebellar pressure cone at autopsy. ${ }^{6}$ Almost all had had one or more lumbar punctures. It seemed clear that the acute syndrome of increased pressure from a mass in the posterior fossa with fatal brain stem compression could unfold without herniation, and in the very cases in which herniation would be most expected. In the following years neuropathologic observations in acute cerebellar hemorrhage corroborated these findings; in some cases there was tonsillar herniation, in others not. There arose the possibility that a cerebellar pressure cone is not a special anatomical means of producing critical pressure on the medulla, but merely represented displacement of cerebellar tissue into the foramen magnum, even affording perhaps some relief from crowding, rather than being especially harmful.

During the same period, there came for pathological study two unusual cases bearing on the matter. Both had sustained vertical fractures of the occipital bone and although intact neurologically were admitted to the hospital for observation. In each case the patient was later found dead in bed, minutes after having been noted to be asymtomatic. At autopsy, both showed large cerebellar herniations $3-4 \mathrm{~cm}$ long with hemorrhagic necrotic tips indicating an age of a few days. In the absence of any other cause, death was attributed to respiratory arrest due to swelling of traumatized cerebellum. At any rate, extreme cerebellar herniation must have been asymptomatic in the day or two before death.

Since on the one hand. death occurs regularly in the absence of a cerebellar cone, and on the other hand a cone may be asymptomatic, it would be difficult to point to a cone as a special mechanism for critically strangling the medulla. The cerebellar herniation with its dire appearance of a plug formed under great pressure and molded into the foramen magnum is perhaps just a sign of an overfilled posterior fossa. This conclusion is not of great practical importance, clinically, since Magnetic Resonance Imaging (MRI) is not often used in critically ill patients and Computed Tomography (CT) may not accurately demonstrate the region. However it may be relevant in pathologic studies since the absence of a pressure cone at autopsy is usually interpreted as evidence that excessive bulk in the posterior fossa was not clinically important in life, a conclusion which may be quite wrong. Also when a herniation is small, measuring for example only 0.5 to $1.0 \mathrm{~cm}$ in height it may be dismissed as insignificant insofar as it is not large enough to have seriously compressed the medulla; again this may be the incorrect inference, the real point being that it is positive evidence of excessive bulk in the posterior fossa. This is not an idle issue considering that pathologic examination may be the clinician's principal guide in the matter.

While on this subject, it may be mentioned that acute posterior fossa-expanding processes (cerebellar hemorrhage, cerebellar infarction, aneurysmal subarachnoid hemorrhage, penetrating wounds, occipital fractures, posterior fossa subdural and extradural hemorrhage) carry as their immediate and main threat, sudden respiratory arrest and death. Intubation may be required as an extreme emergency accompanied by emergency decompression of the posterior fossa. There may not be time for appropriate investigation and proper sterility. A rare condition which usually ends fatally is acute fulminant purulent meningitis in young children. Respiration ceases unexpectedly and autopsy shows a marked cerebellar pressure cone and a thick purulend exudate covering the snug-fitting posterior fosse structures, possibly obstructing the peripontine subarachnoid space. The pathological appearance suggests that prompt decompression might rescue some of these victims.

\section{Upward Cerebellar Herniation}

The same argument advanced for cerebellar pressure cone applies in the case of upward cerebellar herniation. Cerebellar tissue, infarcted or not, is forced upward through the tentorial notch only after all available space in the posterior fossa has been occupied and the expanding content of the overfilled posterior compartment has reached a pressure in excess of the supratentorial pressure. Increased pressure on the brain stem and an actual threat of respiratory embarrassment must have preceded the herniation, which might be construed as beneficial in that an escape of some content occurred. It might be contended that the herniated tissue formed a focal ring or collar of compression, thereby constituting a special mechanism for causing damage to the brain stem over and above that already present, but the clinical course is the same regardless of the ring; there is no evidence the pons is more vulnerable than the medulla. The upward herniated tissue could conceivably contribute to blockage of the pontomesencephalic subarachnoid space giving rise to hydrocephalus, but cerebrospinal fluid (CSF) circulation in the posterior fossa will have already been compromised. A focal hemorrhage in the supero-medial cerebellum has been known to compress the aqueduct locally, precipitating hydrocephalus, but this would not fall in the category of herniation.

The claim is being made that upward cerebellar herniation is an incidental late by-product, a harmless tell-tale, of increased posterior fossa content under diffuse elevated pressure, and not a special instrument for adding to the damage by throttling the brain stem.

\section{ACute temporal Lobe (OR UnCal) Herniation}

When the content of one hemicranium is massively increased acutely, as in intracranial hemorrhage, cerebral infarction with swelling, subdural or extradural hemorrhage, penetrating head injury, etc., the only available direction of expansion after displacing the nearby subarachnoid and ventricular fluid and some venous blood, is towards the opposite hemicranium via the subfalcial window. Structures on either side of the midline are displaced laterally toward the normal hemisphere. The more anterior structures are relatively free to move and horizontal shifts of the pliant septum pellucidum of $2-3 \mathrm{~cm}$ are common. So great are the displacing forces that frequently the falx is strikingly bowed. In the region of the tentorial opening however, little leeway exists for normally there is only $1 \mathrm{~mm}$ or so of space on each side between the midbrain and the rigid tentorial edge. ${ }^{7}$ Lateral displacement therefore cannot be tolerated and a shift from the midline of a few mm results in the midbrain being displaced and compressed against the contralateral unyielding tentorial edge, causing ischemia and structural damage within the brain stem.

On CT, the pineal calcification normally lies in the midline $12-14 \mathrm{~mm}$ superior to the plane of the tentorial opening. It is centered directly above the tegmentum of the midbrain which lies within the tentorial opening. In patients who are becoming 
stuporous with acute unilateral expanding conditions, the pineal calcification is often displaced $6 \mathrm{~mm}$ or more from the midline, that is, the midbrain can be safely displaced only a few millimeters while $12-14 \mathrm{~mm}$ superiorly the third ventricle shifts $6 \mathrm{~mm}$ or more. This gives a clear idea of the compression and angulation the relatively immobile upper brain stem must undergo in the face of the displacing force. (For the sake of brevity in repeatedly referring to the process of brain stem compromise secondary to an acute hemispheric mass lesion, it will here be termed "the acute hemispheric-brain stem syndrome," "the acute syndrome" or "the acute unilateral syndrome". "Acute" refers to a course of up to 24 hours.)

The brain stem complications under discussion constitute the gravest of neurological emergencies as the process unfolds in minutes or hours. The crux of the problem is largely mechanical in nature, thereby offering a favorable chance for surgical management. Particularly vulnerable are patients under the age of 50, especially children. In this younger age group brain atrophy is minimal and the ventricular system is small, with the result that the capacity to adapt to or compensate for an increase in the intracranial content is limited, the intracranial pressure rises and the situation quickly reaches fatal proportions. How severely the brain stem can be compressed and for how long before the point of irreversible damage is reached, are fundamental questions.

In 1921 Meyer $^{2}$ described the presence at autopsy, of temporal lobe herniation in a series of brain tumor cases. The most medial rim of the temporal lobe on the side of the mass was displaced medially to overhang the tentorial edge, forming a sharply defined elongated mass lodged between the tentorial edge and the midbrain within the tentorial opening. Following Meyer, several authors described the presence of temporal lobe or uncal herniation at autopsy in brain tumor cases. Almost always there was the implication that the herniated tissue acted as a wedge to compress the midbrain within the tentorial opening, or in cases of bilateral temporal lobe herniation, to strangle the midbrain. ${ }^{8-11}$ Historically it is notable that all of these studies were made before discovery of the brain stem reticular activating system and the role of brain stem damage in the production of coma. ${ }^{12}$ The herniation was regarded as a specific mechanism by which the brain stem sustained damage. This concept which was derived from pathological studies of chronic tumor cases at an advanced stage still persists today and has been adopted more or less as entirely applicable to events in acute cases. As the syndrome evolves, the pronouncement routinely is "He's herniating". At autopsy it is not easy to evaluate lateral shift of midline structures or the position of the pineal body. In the studies referred to, the pineal position was not recorded in life or at postmortem. The classical rules that pertain to chronic cases may not hold for the acute syndrome and in what follows the gradual development of that point of view will be traced in some detail.

There is another type of herniation at the tentorium referred to as "transtentorial herniation" in which, as the result of a supratentorial mass lesion, the brain stem and the lower thalamus are displaced downwards en bloc through the tentorial opening and into the posterior fossa. This is at present a controversial subject and will be discussed separately.

While studying the significance of cerebellar pressure coning as already described, ${ }^{6}$ the dicta concerning temporal lobe herni- ation also came in for re-examination, particularly as they pertained to the acute syndrome. A number of observations suggested that the issue was not entirely cut and dried.

1) It had been noted at autopsy of hyperacute fatal cases where survival was brief, that occasionally temporal lobe herniation was missing, although midline structures were displaced laterally and the brain stem showed secondary hemorrhages. These were reliable observations in cases with a classical clinical course of progressive stupor and fixed dilatation of first the ipsilateral pupil, then the other. While such cases were not common, they were important in proving that temporal lobe herniation was not essential.

2) It was common clinical experience in typical cases of the acute hemispheric-brain stem syndrome that the prompt administration of a hyperosmolar solution would dramatically reverse the clinical course on one or more occasions temporarily and persistently if definitive therapy could be introduced. It seemed unlikely that a "wedge" of herniated tissue could be retracted repeatedly from the position into which it had been compressed between the tentorial edge and the midbrain. At autopsy the tentorial groove along the medial temporal lobe can be deep and sharply demarcated. Recovery of ipsilateral pupillary function was thought to indicate that the pressure of the herniation on the third nerve had been lifted. During neurosurgical procedures, untimely acute herniation of brain tissue through dural openings is not easily reversed, if at all. Inferences concerning the reversibility of temporal lobe herniation may have been drawn too readily.

3) The view that third nerve compromise in the form of a dilated ipsilateral pupil is an early sign of herniation was difficult to reconcile with clinical experience that, in many acute cases, the contralateral pupil also becomes dilated and fixed in a matter of minutes. The interpretation that dilatation of the first pupil represents pressure on or stretching of the ipsilateral oculomotor nerve, leaves quite unexplained dilatation of the second pupil. Since the pupillary dilatation on the second side has the same characteristics as the first side, doubt is cast on the hypothesis about pressure on the peripheral third nerve. The initial pupillary dilatation seemed to be brought about by an imbalance more delicate than that associated with mechanical stretching of a nerve. Also the ipsilateral eyeball is almost never deviated laterally as would be expected with a peripheral oculomotor palsy. It also happens sometimes that the contralateral pupil dilates before the ipsilateral. The idea that descent of the posterior cerebral artery which has been described as a late event, stretches the third nerve is inconsistent with the occurrence of pupillary dilatation early in the process. Herniation causes compression of the posterior cerebral artery with resultant infarction in the distal territory. This also is a late event, whereas herniation is held to occur early. Infarction should also be expected early. The occurrence of pupillary dilatation as an early sign leaves unaccounted for the $3-24 \mathrm{~h}$ of progressive abulia and stupor that precede pupillary dilatation. While an intra-axial disturbance as the cause of third nerve dysfunction is favored here, a role for extra-axial damage cannot be dismissed. Acute lateral shift of the midbrain could be sufficient to cause injurious stretching of the extra-axial third nerve.

4) Individual cases were encountered that were of interest regarding herniation. a) A 12-year-old boy became increasingly drowsy after a fall from his bicycle. In $8 \mathrm{~h}$ he was comatose, 
with fixed dilated pupils and a respiratory rate of 105 per minute. Emergency craniotomy revealed a bilateral frontal extradural hemorrhage evacuation of which restored the patient to normal. Was a herniation reversed? b) A 24-year-old man developed a headache after striking his head on a car door. Neurological examination $18 \mathrm{~h}$ later was normal. The patient demonstrated his balance by performing the feat of "standing on his hands" before the assembled doctors. Surprisingly angiography showed a $2.5 \mathrm{~cm}$ thick subdural hemorrhage which was immediately removed surgically. After the operation the patient failed to recover from the anesthetic and remained stuporous. Re-angiography revealed a large subdural hemorrhage on the opposite side. Evacuation resulted in prompt recovery. Large bilateral subdural hemorrhages without midline shift had been virtually asymptomatic. It was concluded that removal of one subdural hemorrhage allowed an acute midline shift that was reversed by surgery. Invoking the rapid formation and reduction of a temporal lobe herniation seemed unwarranted. c) An elderly woman who seven years before had suffered a severe stroke with a persistent hemiplegia developed a large hypertensive hemorrhage in the other hemisphere and lingered in stupor for three weeks. Autopsy disclosed the recent hemorrhage along with a large recent temporal lobe herniation $1 \mathrm{~cm}$ wide which had caused no obvious compression of the midbrain. The explanation for this lay in the presence of marked atrophy of the opposite cerebral peduncle secondary to the old stroke. Atrophy allowed harmless displacement of the midbrain with possibly the temporal lobe merely settling into the widened ambient cistern. The appearance did not suggest that herniation had forced the displacement.

Although these 4 categories of reservations concerning the significance of temporal lobe herniation were substantial, casting further light on the process would obviously require more convincing data. Pneumoencephalography and angiography ${ }^{13}$ demonstrated the presence of herniation in tumor cases but did not provide information about the dynamics of events at the tentorium in acute cases.

5) The introduction of high quality CT scanning in the 1970 s brought the great advantage of allowing visualization of normal and pathologic anatomy. Also pineal calcification was demonstrable in 83 per cent of persons over the age of $30 .^{14}$ Osborn $^{15}$ and Stovring ${ }^{16}$ described widening of the ipsilateral ambient cistern early in some cases of the acute syndrome, a finding in keeping with the suggestion that displacement of the midbrain precedes temporal lobe herniation. At a later stage, tissue was shown at the widened cistern consistent with herniation. However, the presence of the uncus at or just above the plane of the tentorial opening is to be expected, since $2-5 \mathrm{~mm}$ of the uncus normally overhang, the tentorial edge. Demonstration of downward displacement through the opening is difficult to display by CT because "partial averaging" makes precise relationships uncertain. The CT observations referred to were made in axial views, not the most favorable plane for visualization of actual herniation. The position of the pineal body was not commented on.

When attention was directed to the position of the pineal calcification it was soon noted that it was laterally displaced from the midline $6 \mathrm{~mm}$ or more. On the basis of the assembled information it was postulated that, in the acute syndrome, the mechanism of midbrain damage at the tentorium was compres- sion by lateral displacement of midline structures with herniation playing no part in the early crucial stages. ${ }^{5.6 .17}$ Temporal lobe herniation, it was suggested, represents a late stage, probably irreversible and as far as treatment is concerned, an irrelevant epiphenomenon. It seemed possible that initially the midbrain was displaced laterally and tissue of the temporal lobe was more or less pressed into the space created in the ambient cistern, rather than the herniation constituting a special instrument or device for exerting critical mechanical pressure on the midbrain. In fact, herniation is usually not a wedge but a gently rounded protrusion less than $5 \mathrm{~mm}$ thick, conforming to the widened ambient cistern and seemingly incapable of exerting leverage. Tissue acting as an entering wedge that is gradually producing increasing displacement of the midbrain would be expected to be fungiform rather than having a rounded, flat contour. Emphasizing and focussing on herniation rather than lateral shift had been an error. Failure of severing the ipsilateral tentorium surgically would have a simple explanation; it was the opposite tentorial crus that was the limiting factor. ${ }^{18}$

Support for this formulation was provided by Ropper's study ${ }^{19}$ showing that deterioration of alertness in the acute syndrome paralleled lateral displacement of the pineal calcification - alert $0-3 \mathrm{~mm}$, drowsiness $3-4 \mathrm{~mm}$, stupor $6-8.5 \mathrm{~mm}$, coma $8-13 \mathrm{~mm}$. These findings were confirmed by Ross et al. ${ }^{20}$

What was still needed were coronal views of the region of the tentorial opening which would show unequivocally whether or not medial temporal lobe tissue had descended below the tentorial plane. In four typical acute cases we were able to reconstruct coronal views from $\mathrm{CT}$ axial views of the posterior fossa. The studies were made within one to four hours of the pupils becoming dilated and fixed. Two patients had hypertensive cerebral hemorrhages and two had unilateral subdural hematomas. In each case the midbrain was flattened against the opposite tentorial edge, the ipsilateral ambient cistern was widened and empty and no evidence of temporal lobe herniation was present (Figure 1). The septum pellucidum was displaced $25-30 \mathrm{~mm}$. It should be pointed out that generally basal structures are not easily identified because of displacement, distortion and effacement of the normal CSF cisterns.

There has been only one opportunity to study an acute case with MRI. A 63-year-old woman with atrial fibrillation suddenly developed hemiplegia as a result of an embolus to the right middle cerebral artery. Thirty-two hours from the onset she was responsive and conversant, although hemiplegic. An MRI was made at 37 hours (23:30). Seven hours after the MRI (07:00) a physician found the patient comatose with the right pupil dilated and fixed. It is suspected that the MRI was fortuitously carried out while the patient was in the early stages of brain stem damage. Assuming the pupil was normal at the time of scanning, the state might be termed "impending acute syndrome". In this light the MRI was of considerable interest. Axial views showed a swollen infarcted right hemisphere with the septum pellucidum displaced $25 \mathrm{~mm}$ to the left, the $3 \mathrm{rd}$ ventricle 5.5 $\mathrm{mm}$ and the aqueduct $5.5 \mathrm{~mm}$. Twenty-four $1 \mathrm{~mm}$ thick axial sections of the midbrain in the region of the tentorium were available as well as $2 \mathrm{~mm}$ thick coronal reconstructions. It could be established with reasonable certainty that the right ambient cistern was widened without evidence of herniation of the medial temporal lobe (Figure 2). A CT scan at the time of the pupillary dilatation showed the same midline displacement as the MRI 10 hours before. 


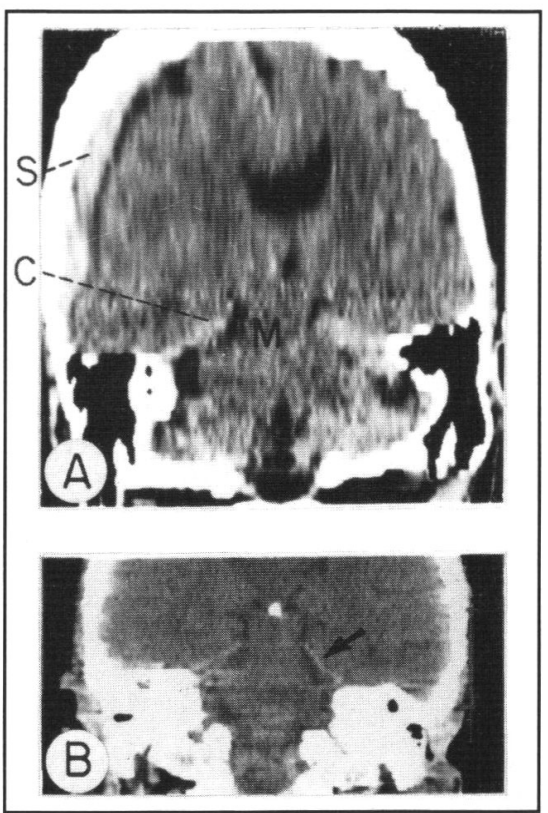

Figure I: A. Representative coronal reconstruction of 3 mm-thick axial $C T$ views to show midbrain at the tentorial opening. An acute rightsided subdural hemorrhage (S) of 6 hours duration has caused coma and a fixed dilated right pupil. Midline structures are displaced to the left, the left side of the midbrain $(M)$ is flattened, the right ambient cistren $(C)$ is widened (black area) and no evidence of a right temporal lobe herniation was present on any of the five coronal sections through the midbrain (see text). B. Comparable normal CT image. Arrow indicates ambient cistern.

Although this additional negative evidence is based on a small number of cases it strengthens the view that temporal lobe herniation is not part of the acute process.

\section{Transten'torial Herniation}

The deliberations concerning temporal lobe herniation have been complicated or at least colored by the widely accepted view that transtentorial herniation is always an accompaniment. According to this tenet, which was introduced by Scheinker, ${ }^{3}$ in the acute hemispheric-brain stem syndrome the posterior-inferior thalamus and the midbrain are displaced vertically downwards through the tentorial opening. Scheinker ${ }^{3}$ was of the opinion that downward movement was more significant than temporal lobe herniation.

Howell ${ }^{21}$ presented pathological data supporting the idea of downward displacement which in its most severe form caused backward buckling of the midbrain. Plum and Posner ${ }^{22}$ made transtentorial herniation the pathological basis of their "central syndrome", the most common clinical picture associated with the acute hemispheric-brain stem process. Downward displacement can be found in autopsy studies but most likely it is a late terminal development in patients maintained on ventilators and there has been no demonstration of its occurrence as an early feature.

In approaching this problem using $\mathrm{CT}$, it was noted that in standard axial views, the pineal calcification normally lies in the same horizontal plane as or just above the choroid plexus calcifications on each side. ${ }^{6}$ Assuming that downward displacement of the midbrain would be associated with a similar downward

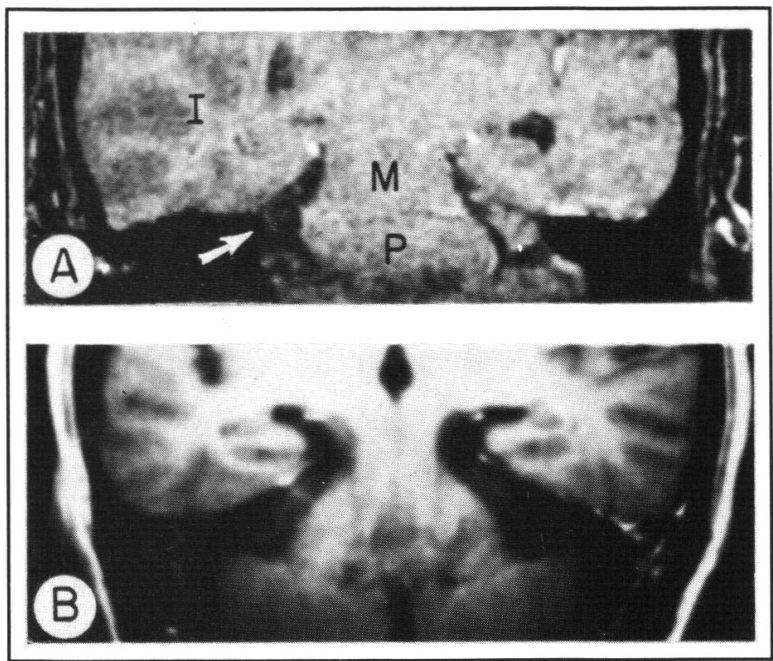

Figure 2: A. Representative comnal reconstruction of $241 \mathrm{~mm}$-thick axial MRI sections of the midbrain in the region of the tentorial opening. The right hemisphere is infarcted (I), the right temporal horn is almost obliterated, the aqueduct is displaced $5.5 \mathrm{~mm}$ to the left (not shown) and the right ambient cistern (arrow) is wider compared with the left, without evidence of herniation of the right medial temporal lobe. $M=$ Midbrain, $P=$ Pons (see text). B. Comparable normal comnal (unreconstructed) MR image.

displacement of the pineal calcification which normally lies 12 $14 \mathrm{~mm}$ superiorly, there was the prospect that here was a method of determining vertical movement of the upper brain stem. Downward movement of the pineal calcification would bring it out of the plane of the plexus calcifications. In the acute unilateral syndrome the pineal calcification would be compared with the plexus calcification on the non-involved side whose position should not be altered, whereas on the acutely involved side displacement might be expected. Using these markers as an index of vertical downward displacement of the pineal calcification, 20 cases of the acute syndrome were studied at the stage of coma with fixed pupillary dilatation on one side or both. ${ }^{6}$ In all cases the pineal calcification remained in the same plane as the choroid plexus calcification on the non-involved side. In other words there was no evidence of transtentorial herniation (Figure 3).

The 3-calcification method drew criticism on the grounds that small pineal movements might not be detected because of the rather large size and variable shape of the calcifications. There have been many cases, however, in which CT studies could be made early and late, the first study serving as a reliable reference for any slight later change. Also it has been claimed that the pineal calcification may not be mobile owing to its position within an arachnoidal meshwork. Contradicting this is the lateral mobility of the pineal, as well as the fact that in CT studies made very late in the hours just before death in similar cases of the acute syndrome, pineal descent of $1 \mathrm{~cm}$ or more has been found, demonstrating that pineal calcification is capable of moving downward. Moreover this descent confirms suggestions that the autopsy studies showing downward transtentorial herniation represented a very late preterminal development and did not reflect early relevant events. In most instances however the pineal calcification is not displaced downwards even at a late stage. 


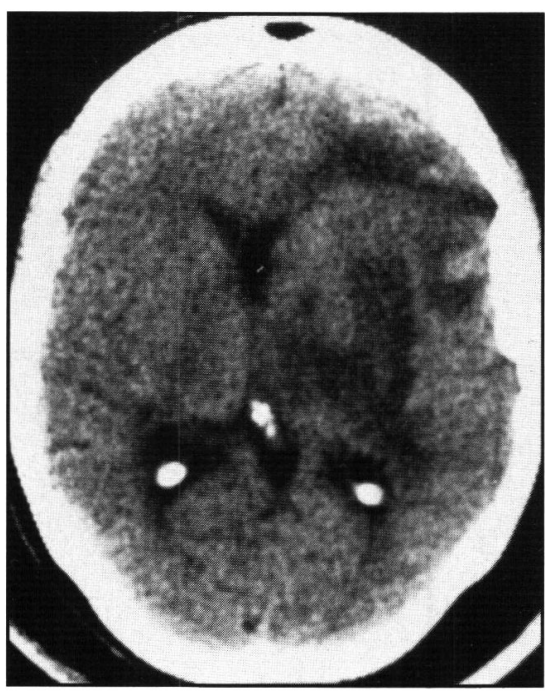

Figure 3: CT scan to illustrate use of the 3-calcification method of detecting vertical pineal movement in a case of massive left hemispheric infarction. The pineal calcification is displaced $7 \mathrm{~mm}$ to the right, but has remained in the same plane as the choroid plexus calcification on the right (normal) side.

MRI should be particularly suited to detection of vertical displacement of the brain stem. However acute hemisphericbrain stem cases are critically ill and requiring ventilators, which usually precludes an MRI examination. MRI hạs been used in a series of patients less acutely ill with an assortment of intracranial conditions, to measure vertical downward movement of the brain stem at the tentorium. ${ }^{23}$ The amount of displacement did not correlate with the clinical status. Using as a brain stem marker the upper entrance into the aqueduct, downward displacement in 18 supratentorial cases varied from 3.2 to $15.5 \mathrm{~mm}$. Patients could be stuporous with a displacement of 4 $\mathrm{mm}$, or close to normal neurologically with a displacement of $11 \mathrm{~mm}$. After cranial surgery a patient's condition could improve without a change in the downward displacement. Although displacement may have been associated with a patient's neurological state, it was not shown to be causative. The study did not include patients with the acute hemisphericbrain stem syndrome. A shortcoming of the method of measurement is that in cases with lateral displacement of the midbrain, the midline landmarks are lost. Tonsillar herniation was also measured in this study, ${ }^{23}$ cerebellar descent below the line of the foramen magnum being used as the gauge. Tonsillar herniation varied from zero to $13.6 \mathrm{~mm}$ but did not correlate with brain stem descent at the tentorium and with or without concomitant brain stem descent was well tolerated and free of any signs of impending disaster. These observations agree with the autopsy findings of Schwarz and Rosner ${ }^{9}$ that cerebellar pressure coning occurred in only 20 per cent of cases of temporal lobe herniation and severity at the two sites was not correlated.

Ropper ${ }^{24}$ described a comatose patient with bilateral dilated fixed pupils whose aqueductal entrance was not displaced downward despite the presence of a large ipsilateral temporal lobe herniation. In our acute case described in the previous section, sagittal MRI showed the aqueductal entrance elevated $3 \mathrm{~mm}$. Further evidence contradicting the idea of symptomatic descent of the brain stem was furnished by MRI studies in cases of low spinal fluid pressure. Descents of $7-14 \mathrm{~mm}$ were asymptomatic except for headache. ${ }^{25}$

It can be concluded with fair certainty that transtentorial herniation is not a factor in the acute syndrome, especially in the early crucial reversible phase.

\section{The Dynamics of Events in the Region of the Tentorium}

On the basis of the findings so far, the dynamics of events taking place along the midline in the acute syndrome may be as follows. An acute hemispheric mass, such as a hypertensive hemorrhage, on attaining a size of $3.4 \mathrm{~cm}$, treks toward the ipsilateral ventricle, following which the midline structures are displaced toward the opposite side through the midline subfalcial window. The subarachnoid space is effaced early, both at the base and over the affected hemisphere. Rapidly increasing brain edema and a further rise in the intracranial pressure compound the threat. Hemorrhages almost never rupture outwards through the cortex to reach the subarachnoid space; the cortex supported by the skull is an impenetrable barrier.

The subfalcial window is roughly $9.5 \mathrm{~cm}$ long and $5 \mathrm{~cm}$ vertically. The falx above is also $5 \mathrm{~cm}$ in height making the window about one-half the sella turcica-vertex distance. Normally the ventricular system occupies the window flanked inferiorly on each side by the basal ganglia and the thalami. The inferior boundary of the subfalcial window is formed in its anterior half by the ethmoid and sphenoid bodies including the sella turcica. Its posterior half is occupied by the tentorial opening which at that level measures $5.5 \mathrm{~cm}$ long by $3.0 \mathrm{~cm}$ wide, ${ }^{26}$ and encloses the middle part of the midbrain. The plane of the tentorial opening inclines $30^{\circ}$ upward.

The plane of the subfalcial window is sagittal or vertical and is about $50 \mathrm{~cm}^{2}$ in area. The plane of the tentorial opening is roughly axial and $18 \mathrm{~cm}^{2}$ in area. Presumably the displacing forces created by a hemispheric mass are mainly horizontal and directed towards the bodies of the lateral ventricles and the central part of the large subfalcial window. Any vector of force displacing the brain downwards through the axially disposed tentorial opening would be acting at a disadvantage as it would be almost at a right angle to the main displacing force, and directed towards a smaller aperture occupied anteriorly by the midbrain. The tentorial surfaces slope upwards toward the opening, in this way opposing a downward thrust. The brain, having the properties of a plastic, transmits forces by vector contiguity, not uniformly in all directions as does a fluid. ${ }^{27}$ The failure of the brain to herniate downwards through the tentorial opening in the early phase of the acute syndrome becomes understandable.

The force displacing central structures laterally towards the uninvolved side may carry the thin, freely movable septum pellucidum separating the two ventricles $2-3 \mathrm{~cm}$ from the midline. Superiorly, the corpus callosum is displaced, carrying with it the adjacent cingulate gyrus beneath the falx. Further posteriorly the thalami and the interposed third ventricle are displaced, but less far than the septum pellucidum. The third ventricle is greatly compressed, even to near obliteration. The pineal body lies between the thalami in the plane of the third ventricle about $5 \mathrm{~mm}$ anterior to the posterior margins of the pulvinars. Here the pineal body is $12-14 \mathrm{~mm}$ above the plane of the tentorial opening and centered directly over the midbrain 
tegmentum which lies within the opening. The horizontally oriented displacing force on the thalami, which must be considerable judging from the lateral displacement of the pineal body, is acting rather low in the posterior half of the subfalcial window. A displacement of $10 \mathrm{~mm}$ would carry the pineal body laterally almost to the plane of the tentorial edge, making descent through the tentorial opening impossible and theoretically severing half the midbrain if it was similarly displaced.

The restricted lateral movement of the midbrain in contrast with the freer movement of midline structures just above the tentorium increases the distortion of vital structures in the supratentorial zone. The complex mechanical derangements that result can only be inferred. The midbrain is compressed against the tentorial edge and bears the brunt of the insult (dilated fixed pupils). The associated coma results from dysfunction in the ascending reticular activating system at the midbrain level, as well as in displaced more rostral structures. Immediately above the tentorium the central structures are subject to shearing, stretching and rotational forces. Many penetrating arteries to the midbrain are torn apart within the parenchyma, giving rise to secondary brain stem hemorrhages. ${ }^{28}$ It is easy to believe that the major force displacing thalami, midbrain and pons is generated by the leverage of the broad medial aspect of the swollen hemisphere. Any local increment of force on the midbrain attributable to a small uncal herniation would be inconsequential. One might even conceive of herniation as providing a slight decrease in the laterally-directed, injurious displacing force. An appreciation of the magnitude of the abnormal forces involved is difficult to gain. The readiness with which a dilated fixed pupil regains normal function upon the administration of mannitol suggests a rather gentle displacing force combined with great vulnerability of the tissue of the brain stem to physical deformation. If the pressure with which the midbrain is compressed against the tentorial edge were great, the Kernohan notch should regularly be deeper and more destructive. The impression often conveyed by the pathological picture of a process of considerable intensity, is probably misleading. Would severing the contralateral crus of the tentorium afford relief from compression? Granting that were feasible, there is the prospect that brain stem distortion might be decreased but damaging displacement of the hypothalamus be increased.

Compression of the aqueduct or the third ventricle, or both, will obstruct CSF flow downwards, further increasing the supratentorial pressure, as CSF is produced at a rate of $10 \mathrm{ml} / \mathrm{hr}$ in each lateral ventricle. Obstruction at the tentorial opening will hinder the upward passage of the CSF from the posterior fossa. In this regard Munro and Sisson ${ }^{18}$ in surgically exploring cases of "herniation" made stab incisions in the tentorium; CSF gushed upwards out of the posterior fossa.

Having decided that the mechanism of brain stem signs in the acute hemispheric-brain stem syndrome is lateral displacement of midline structures rather than temporal lobe herniation, the question might be asked, Is this not hairsplitting, especially since any emergency intervention would be the same in either case? Visualizing herniation when it is not a factor must be disadvantageous. Correct visualization of the dynamic details of the acute process could be important in thinking about the problem and devising timely therapeutic strategy. In interpreting the results of therapy it may well make a difference. The two processes are related but lateral displacement is the prime mover. If in acute cases the proposition of herniation as a special mechanism of damage is incorrect, why perpetuate it? Use of correct terminology is a worthy aim on its own.

\section{The Bilateral Hemispheric-brain Stem Syndrome; The ACUTE BILATERAL SYNDROME}

These terms refer to the occurrence simultaneously of acute cerebral mass lesions bilaterally as in cerebral edema, cranial trauma, bilateral subdural hematomas, acute aneurysmal hemorrhage with abundant subarachnoid blood, bilateral tumors, bilateral infarctions, acute purulent meningitis, viral encephalitis, increased intracranial pressure, etc. Again emphasis is on processes that are quite symmetrical and unfolding acutely rather than in weeks or months. The effects are vastly different from the unilateral form, probably because with the two hemispheres equally swollen, tissue pressures will be approximately equal in the two hemispheres with little or no tendency to lateral displacement of midline structures, including the midbrain and pineal body. Temporal lobe herniation would not be expected at least where the midbrain is broadest and the ambient cisterns are only $1 \mathrm{~mm}$ wide. If this interpretation is correct, it furnishes strong indirect evidence favoring lateral shift at the tentorium as the mechanism of brain stem damage in the acute unilateral syndrome.

As the supratentorial swelling and crowding increase and the CSF is displaced from the subarachnoid space and the ventricular system, the medial temporal lobes become apposed to the midbrain resulting in what Vincent et al. ${ }^{8}$ called strangulation of the brain stem. These authors found bilateral temporal lobe pressure cones at autopsy in chronic brain tumor cases where there is time for molding of the medial temporal lobes to take place and the midbrain becomes compressed and narrowed in an anterior-posterior direction. It is my experience that in the acute bilateral syndrome, bilateral temporal lobe herniation does not take place. By CT and at autopsy the medial temporal lobes "hug" the midbrain and for this, the term "bitemporal brain stem compression" (BBC) has been suggested..$^{29}$ As the "squeezing" force increases the midbrain becomes ischemic, resulting in a clinical syndrome resembling that of the acute unilateral syndrome. Shallow molding of the medial temporal lobes bilaterally into the tentorial opening posterior to the midbrain is part of the process and may contribute in a minor way to the compression. The patient becomes abulic, drowsy and stuporous as first the pupil on one side dilates, then the other, followed by bilateral decerebrate posturing. Secondary brain stem hemorrhages are rare compared with their frequency in the acute unilateral syndrome. This could have important clinical implications in that the midbrain is not irreparably damaged as promptly as in the unilateral syndrome where it is stretched, distorted and compressed against the tentorial edge. This means that the situation does not become hopeless as early in the process and quick therapeutic intervention may have a chance. Paralleling these events the pineal calcification should remain within 1 or $2 \mathrm{~mm}$ of the midline. It is my experience, ${ }^{6}$ using the 3 calcification method of gauging downward displacement of the pineal calcification, that downward transtentorial herniation of the brain stem does not happen in this situation although 
others have described it. ${ }^{22}$ It could develop very late in patients maintained on ventilators when the descent is found at autopsy in the form of brain stem buckling. However, even several hours into the stage of coma it has generally not been present in our cases. As in the unilateral syndrome compression of the aqueduct and jamming of the perimesencephalic cisterns will block the circulation of CSF with effects that depend on which avenue becomes obstructed first. When the aqueduct remains open, pressure in the posterior fossa will rise inordinately. The proposal has been made that in the acute bilateral syndrome associated with ruptured aneurysm, in which all efforts to reduce intracranial pressure are failing, a large bilateral decompressive craniectomy be undertaken as an emergency. ${ }^{32}$ The pathological anatomy will vary from case to case and the recommendations made will need tempering accordingly.

\section{COMMENT}

The salient observations and arguments have been discussed in each section and little is left to be added. There is one important matter however which must be examined more closely and that is the danger of lumbar puncture in such cases. It is well appreciated and correctly so, that in chronic hemispheric expanding processes, perimesencephalic molding of the medial part of one or both temporal lobes occurs, producing a degree of obstruction to CSF flow. In this setting lumbar puncture with a drastic reduction of the CSF pressure may precipitate fatal acute brain stem compression. If this response occurred early in the acute process, it would offer evidence in favor of herniation as a damaging mechanism. In recent years we have not had an opportunity to make reliable observations in cases of this type. With events unfolding rapidly and the intracranial pressure markedly elevated, the mechanism of any deterioration after a lumbar puncture might be difficult to interpret. In the cases presented in this paper lumbar puncture was not a factor.

Lumbar puncture in cerebellar pressure coning probably carries a similar risk although Smyth and Henderson ${ }^{11}$ reported that in tumor cases with raised intracranial pressure, even pronounced cerebellar herniation did not disturb CSF dynamics. The possible presence of acute marked bilateral hydrocephalus secondary to an overfull posterior fossa would present another complication.

In this paper an attempt has been made to revamp the clinical teachings about brain herniation, particularly in acute cases. Temporal lobe herniation and cerebellar pressure coning have been removed from the category of special instruments for critically aggravating the damage caused by the primary disease condition. They are to be regarded as rather harmless late accompaniments of more lethal intracranial processes - lateral displacement of midline structures in the case of temporal lobe herniation and posterior fossa overcrowding in the case of cerebellar pressure coning. In supratentorial lesions the horizontal position of the pineal calcification becomes pivotal. The general principle also holds true for chronic processes if lumbar puncture is not a complicating factor. There is no reason why molding by itself at the temporal opening or foramen magnum should be construed as a special threat. The phenomenon of bitemporal brain stem compression (BBC) in bilateral acute hemispheric cases has been presented in some detail. The idea of transtentorial herniation as a relevant factor in the early crucial stage of acute mass lesions has not found support. Subfalcial herniation remains an early hazard.

Although the evidence in favor of this new concept is, in a way, compelling its possible shortcomings should be addressed. The very nature of the process, hidden as it is deep in the brain, and its complexity are such that decisive answers are not readily achieved. The study was mainly observational using case material as it happened by chance to become available with the desired clinical and imaging information. The patients were usually critically ill and not candidates for special or repeat studies. Coma cases of different mechanisms served as "controls". The ideas described here were sometimes tested by blindly predicting a patient's clinical state from the appearance of the imaging studies. Cases were not deliberately excluded because their findings disagreed with the general tenet.

Cerebellar pressure coning may have been dismissed on insufficient grounds and in recent years there has not been the occasion to restudy the matter. As for temporal lobe herniation, CT images of the pathologic anatomy in the region of the tentorial opening are often difficult to interpret because of the distortion of structures and failure to identify the tentorial edges. Coronal views are preferable, a requirement that limits the number of suitable cases. More cases studied by MRI or both MRI and CT would be most informative. In patients with bloody CSF or who have received intravenous contrast agent, structures in the region of the tentorium may be outlined more clearly. Unfortunately such cases are uncommon. We did not have an opportunity to image patients before and immediately after receiving mannitol intravenously. But having stated our current views somewhat emphatically others are provided with findings with which to match their own observations.

Whenever asked to suggest a clinical term to replace "herniating", a suitable designation does not spring to mind. One might say the patient is developing the midbrain syndrome, or the central syndrome, or simply, "he's midbraining".

\section{ACKNOWLEDGEMENT}

The support of the Mihara Memorial Foundation, Tokyo is gratefully acknowledged. This paper was the subject of the IV Shelley Chou Lecture in Neurosurgery University of Minnesota, Minneapolis, Minnesota, June 12, 1994.

\section{REFERENCES}

1. Cushing H. Some principles of cerebral surgery. JAMA 1909; 52: 184-195.

2. Meyer A. Herniation of the brain. Arch Neurol Psychiatr 1920; 4: $387-400$.

3. Scheinker IM. Transtentorial herniation of the brain stem; a characteristic clinicopathologic syndrome; pathogenesis of hemorrhages in the brain stem. Arch Neurol Psychiatr 1945; 53: 289-298.

4. LeBeau J. L'oedème du Cerveau. Paris, Recht 1938.

5. Fisher CM. The neurological examination of the comatose patient. Acta Neurol Scand 1969; 45 (Suppl 36): 1-56 p.52.

6. Fisher CM. Acute brain herniation - a revised concept. Semin Neurol 1984; 4: 417-421.

7. Elguera M. Transtentorial herniation. In: Finney LA, Walker AE, eds. Springfield III: Charles C Thomas, 1962.

8. Vincent $C$, David M, Thiébaut F. Le cône de pression temporal dans les tumeurs cérébrales. Rev Neurol 1936; 65: 536-545.

9. Schwarz GA, Rosner A. Displacement and herniation of the hippocampal gyrus through the incisura tentorii: a clinicopathologic study. Arch Neurol Psychiatr 1941; 46: 297-321. 
10. Jefferson G. The tentorial pressure cone. Arch Neurol Psychiatr 1938; 40: 857-876.

11. Smyth GE, Henderson WR. Observations on the cerebrospinal fluid pressure on simultaneous ventricular and lumbar punctures. J Neurol Psychiatr 1938; 1: 226-238.

12. Magoun $\mathrm{HW}$. An ascending reticular activating system in the brain stem. Arch Neurol Psychiatr 1952; 67: 145-154.

13. Azambuja N, Lindgren E, Sjögren SE. Tentorial herniations. Acta Radiol (Stockh) 1956; 46: 215-223, 223-231, 232-239.

14. Macpherson P, Matheson MS. Comparison of calcification of pineal, habenular commissure and choroid plexus on plain films and computed tomography. Neuroradiology 1979; 18: 67-72.

15. Osborn AG. Diagnosis of descending transtentorial herniation by cranial computed tomography. Radiology 1977; 123: 93-96.

16. Storing J. Descending tentorial herniation: findings on computed tomography. Neuroradiology 1977; 14: 101-105.

17. Fisher $\mathrm{CM}$. Observations concerning brain herniation. Ann Neurol 1983; 14: II0 (abst.)

18. Munro D, Sission WR jr. Hernia through the incisura of the tentorium cerebelli in connection with craniocerebral trauma. $\mathrm{N}$ Engl J Med 1952; 247: 699-708.

19. Ropper AH. Lateral displacement of the brain and level of consciousness in patients with an acute hemispheral mass. $\mathrm{N}$ Engl J Med 1986; 314: 953-958.

20. Ross DA, Olsen WL, Ross AM, Andrews BT, Pitts LH. Brain shift, level of consciousness, and restoration of consciousness in patients with acute intracranial hematoma. J Neurosurg 1989; 71 : 498-502.
21. Howell DA. Longitudinal brain stem compression with buckling: some further observations on a form of upper brain stem compression with intracranial space occupying lesions and brain swelling. Arch Neurol 1961; 4: 572-579.

22. Plum F, Posner JB. The Diagnosis of Stupor and Coma. 3rd ed. Philadelphia, FA Davis 1980.

23. Reich JB, Sierra J. Deck MDF, Plum F. MRI description and clinical correlation of dynamic upward and downward transtentorial and foramen magnum brain herniation. Ann Neurol 1993; 33: 159-170.

24. Ropper AH. Syndrome of transtentorial herniation: is vertical displacement necessary? J Neurol Neurosurg Psychiatry 1993; 56: $932-935$

25. Pannullo SC, Reich JB, Krol G, Deck MDF. Posner JB. MRI changes in intracranial hypotension. Neurology 1993; 43: 919 . 926.

26. Sunderland $S$. The tentorial notch and complications produced by herniations of the brain through that aperture. Br J Surg 1958; 455: $422-438$.

27. Hakim S, Venegas JG, Burton JD. The physics of the cranial cavity, hydrocephalus and normal pressure hydrocephalus. Mechanical interpretation and mathematical model. Surg Neurol 1976; 5 : 187-210.

28. Fisher CM. The arterial source of secondary brain stem hemorrhages. J Pathol 1972; 66: 9a (abst).

29. Fisher CM, Ojemann RG. Bilateral decompressive craniotomy for worsening coma in acute subarachnoid hemorrhage. Observations in support of the procedure. Surg Neurol 1994; 41 : 65-74. 